\title{
LIMB SALVAGE IN CRITICAL LIMB ISCHAEMIA IN THROMBOANGIITIS OBLITERANS PATIENTS- REVASCULARISATION- A STUDY
}

\author{
Maruthu Thurai Sambandam¹, Velladuraichi Boologapandian², Amalorpavanathan Joseph ${ }^{3}$
}

${ }^{1}$ Senior Resident, Department of Vascular Surgery, Institute of Vascular Surgery, Madras Medical College, Chennai, T. N., India. ${ }_{2}^{2}$ Assistant Professor, Department of Vascular Surgery, Institute of Vascular Surgery, Madras Medical College, Chennai, T. N., India. 3 Professor, Department of Vascular Surgery, Institute of Vascular Surgery, Madras Medical College, Chennai, T. N., India.

\section{ABSTRACT}

\section{BACKGROUND}

Buerger's disease, pathologically named Thromboangiitis Obliterans (TAO) has for long remained a disease difficult to understand due to the various theories ascribed to its pathogenesis and lack of consensus regarding accurate diagnostic criteria. A significant proportion of our patients in our population present with characteristic features of TAO, except that they have occlusive disease in the medium and larger sized arteries including the femoral and iliac arteries.

This study attempts to present our principles guiding revascularisation in critical limb ischaemia patients with TAO and analyse the surgical outcomes in terms of graft patency, ulcer healing and limb salvage.

\section{MATERIALS AND METHODS}

This is a retrospective observational study. All patients admitted with the clinical diagnosis of TAO from August 2011 to December 2013 (retrospective study) were included and their case sheets were analysed. Salient features were then recorded from these case records including age, clinical presentation and management strategies employed. Patients underwent conservative management, primary amputation or plan for revascularisation. Shionoya criteria were used for the classification of the disease.

\section{RESULTS}

The total no. of revascularisation procedures done were 60. In the planned 60 patients, surgical revascularisation could be accomplished in only 50 patients, while in 10 patients the planned bypass had to be abandoned in view of either poor distal outflow or lack of quality vein. In the operated group of patients, five patients underwent iliofemoral bypass with synthetic graft. Twentyfive patients underwent femoro-popliteal bypass, while twenty patients underwent tibial bypass with autologous vein graft. All patients were operated under regional anaesthesia. Average duration of surgery ranged between 3 - 4 hours. All patients received anticoagulation. Surgical revascularisation resulted in relief of rest pain in thirty six out of the sixty patients in the immediate postoperative period. The average period of hospitalisation was seven days postoperatively. There was no postoperative mortality. At 6 months of follow-up, thirty-two patients showed successful results in terms of surgical outcome with twenty patients having a palpable pulse and twelve patients showed ABI improvement. This recorded a primary graft patency rate of $64 \%$. The thirty-six limbs salvaged recorded a limb salvage rate of $80 \%$. Major amputation was necessitated in nine patients with an amputation rate of $20 \%$. Minor amputation was done on all the salvaged limbs, which included great toe disarticulation in seven patients, other toe disarticulation in nine patients and forefoot amputation was needed in twenty patients (80\%). Post revascularisation complications included major infection ( $\mathrm{n}=1$ ) with graft infection resulting in pseudoaneurysm of the distal anastomosis of the anterior tibial artery despite a successful bypass and a below knee amputation was needed in one patient. Other complications included minor bleeding $(\mathrm{n}=2)$, minor wound infections $(\mathrm{n}=9)$ which were treated conservatively.

\section{CONCLUSION}

TAO continues to be a significant source of limb loss and morbidity. The mean age of all TAO patients with CLI was 35 years. Most patients presented with critical limb ischaemia. Angiographically, majority had femoropoliteal segment occlusion. Suprageniculate disease affects a significant proportion of patients with TAO in our population. All patients with suprageniculate disease have infrageniculate disease as well. Primary amputation was needed in $30 \%$ patients with TAO. Revascularisation could be attempted in 10 - 15\% patients with TAO. Suprapopliteal vessel disease is more amenable for revascularisation with the possibility of a suitable distal vessel unlike infrapopliteal disease, where suitable distal vessels for revascularisation are often absent. Pathogenic mechanisms for initiation and progression of disease at different levels in the arterial tree are different. The different pathogenic and anatomic factors impact the probability of revascularisation and limb salvage.

\section{KEY WORDS}

TAO, Critical Limb Ischaemia, Limb Salvage, Revascularisation, Amputation.

HOW TO CITE THIS ARTICLE: Sambandam MT, Boologapandian V, Joseph A. Limb salvage in critical limb ischaemia in thromboangiitis obliterans patients- revascularisation- a study. J. Evolution Med. Dent. Sci. 2018;7(33):3679-3683, DOI: $10.14260 /$ jemds/2018/826

'Financial or Other Competing Interest': None.

Submission 28-08-2017, Peer Review 31-07-2018,

Acceptance 06-08-2018, Published 13-08-2018.

Corresponding Author:

Dr. Maruthu Thurai Sambandam,

\#53, IV Street, Kuzhandai Ammal Nagar,

Thanjavur-613007, Tamilnadu, India.

E-mail: drsmdurai@gmail.com

DOI: $10.14260 /$ jemds $/ 2018 / 826$

\begin{abstract}
BACKGROUND
Buerger's disease, pathologically named Thromboangiitis Obliterans (TAO) has for long remained a disease difficult to understand due to the various theories ascribed to its pathogenesis and lack of consensus regarding accurate diagnostic criteria.

Thromboangiitis Obliterans (TAO) continues to be an important cause of limb loss and morbidity in our population. Unlike the rest of the world, there has been no respite with
\end{abstract}


regards to the incidence of this disease in our population owing to the ill effects of tobacco abuse, especially beedi smoking and tobacco chewing (Matsushita Metal).

A significant proportion of our patients in our population present with characteristic features of TAO, except that they have occlusive disease in the medium and larger sized arteries including the femoral and iliac arteries. Involvement of proximal vessels is observed in our population, contrary to disease definitions elsewhere in the world and this needs focused research.

The lack of clarity and the importance of the disease has inspired us to conduct this study in order to understand the disease better and evolve strategies to limit limb loss and morbidity in these young patients. Further the fallout of this disease has got far reaching social and economic ramifications owing to the young age and male preponderance of the disease pattern woefully affecting families in the lower socioeconomic strata. Attempts at limb salvage in these patients are vital in bringing hope to these unfortunate patients.

This study attempts to present our principles guiding revascularisation in critical limb ischaemia patients with TAO and analyse the surgical outcomes in terms of graft patency, ulcer healing and limb salvage.

\section{Aim}

To study the feasibility and effectiveness of lower limb revascularisation in patients with critical limb ischaemia, satisfying clinical criteria for TAO, (Ref: $1,2,3$ ) with emphasis on-

- Principles guiding revascularisation in TAO patients with CLI.

- $\quad$ Ulcer Healing.

- Graft Patency.

- Limb Salvage.

\section{MATERIALS AND METHODS}

All patients admitted with the clinical diagnosis of TAO from August 2011 to December 2013 (Retrospective study) were included and their case sheets were analysed. Salient features were then recorded from these case records including age, clinical presentation and management strategies employed. Patients underwent either conservative management, primary amputation or plan for revascularisation. Patients who underwent revascularisation and satisfied the inclusion criteria formed the main study group. Institutional ethical clearance was obtained for the study. Shionoya criteria was used for the classification of the disease (Ref: $4,5,6$ ).

\section{Inclusion Criteria}

- $\quad$ Age of onset of disease $<50$ yrs.

- Tobacco use (current or recent).

- Upper limb involvement.

- Critical limb ischaemia.

- CT angiogram findings of a distal outflow vessel.

\section{Exclusion Criteria}

- Presence of risk factors for atherosclerosis such as Diabetes mellitus, Systemic hypertension, Dyslipidaemia.

- Angiographic features of Atherosclerosis such as proximal vessel involvement, vessel wall calcification.

- Echocardiographic or angiographic evidence of proximal embolic source including cardiac intramural thrombus or proximal arterial aneurysms.
- Laboratory evidence of other vasculitis including raised inflammatory markers (Erythrocyte sedimentation rate and C-reactive protein).

\section{Diagnostic Evaluation in TAO:}

- Confirmation of Tobacco use.

- Ankle brachial index.

- CRP, ESR.

- Serum homocysteinaemia.

- Coagulation profile.

- Autoimmune screen.

- Serologic markers for CREST syndrome.

- Hepatitis B, C, VDRL.

- Duplex and Echocardiography.

- Toxicology screen.

- CT angiogram.

- $\quad$ Biopsy- Unusual locations, Age -> 45.

\section{Protocols Adhered for Handling Vein Segments}

- Pre-operative vein mapping.

- Very gentle dissection.

- Use of slings to handle vessels in place of cotton tape.

- Use of volume expanders- Dextran.

Any crural vessel with at least $10 \mathrm{~cm}$ of patency, preferably in continuation with pedal arch or draining good collateral network was taken as the outflow vessel. Immediate postoperative anticoagulation is the standard practice and anti-platelets with statins were prescribed in the follow-up period. Vitamin $\mathrm{K}$ antagonists was advised in patients with single vessel outflow.

\section{Statistical Analysis}

Statistical analysis was performed using the SPSS software.

\section{RESULTS}

\section{No. of Revascularisation in TA0-60}

- $\quad$ Iliofemoral bypass- 5 .

- Femoropopliteal bypass- 25.

- $\quad$ Tibial bypass- 20.

- $\quad$ Non-reconstructable- 10.

Of all the patients with a clinical diagnosis of TAO, 60 patients with critical limb ischaemia were planned for surgical revascularisation. All patients fitted in with Shionoya's criteria. Their CT angiograms showed a distal bypassable vessel and thus were included in the study. Duplex was done in all these patients, both to study the artery under consideration for bypass as well as to carry out preoperative vein mapping. Surgical revascularisation procedures for TAO $(n=60)$ constituted $17 \%$ of all bypass surgeries $(n=345)$.

In the planned 60 patients, surgical revascularisation could be accomplished in only 50 patients, while in 10 patients the planned bypass had to be abandoned in view of either poor distal outflow or lack of quality vein. Presence of inflammatory thrombus in the distal vessel was quite a significant factor forcing abandonment of the procedure in this group. Of these ten patients, five patients ended up with amputation in the next 3 - 6 months. The other five patients were lost in the follow-up. 
In the operated group of patients, five patients underwent iliofemoral bypass with synthetic graft. Twenty-five patients underwent femoropopliteal bypass, while twenty patients underwent tibial bypass with autologous vein graft. All patients were operated under regional anaesthesia. Average duration of surgery ranged between 3 - 4 hours. All patients received anticoagulation. 25 patients needed blood transfusion because of preoperative low haematocrit.

Surgical revascularisation resulted in relief of rest pain in thirty six out of the sixty patients in the immediate postoperative period. The average period of hospitalisation was seven days postoperatively. Postoperative ICU stay was for one day in these patients with no patient requiring postoperative ventilation. There was no post-operative mortality.

At 6 months of follow-up, thirty-two patients showed successful results in terms of surgical outcome with twenty patients having a palpable pulse and twelve patients showed $\mathrm{ABI}$ improvement. This recorded a primary graft patency rate of $64 \%$. In this group, four patients developed graft thrombosis and were taken up for intervention in the immediate postoperative period. Graft thrombectomy restored pulse in two patients. The reason for graft thrombosis was technical in nature. One patient had problem in the tunnelling of graft, while the other patient had a distal anastomotic failure which was corrected. In the other two patients, graft thrombectomy failed.

Four patients recorded same ABI post revascularisation, but their limbs were salvaged. The thirty-six limbs salvaged recorded a limb salvage rate of $80 \%$.

Major amputation was necessitated in nine patients with an amputation rate of $20 \%$. (Above knee amputation- 6 and below knee amputation- 3). Minor amputation was done on all the salvaged limbs which included great toe disarticulation in seven patients, other toe disarticulation in nine patients and forefoot amputation was needed in twenty patients (80\%). Ulcers healed in all these patients with split skin grafting.

Post revascularisation complications included major infection $(n=1)$ with graft infection resulting in pseudoaneurysm of the distal anastomosis of the anterior tibial artery, despite a successful bypass and a below knee amputation was needed in one patient. Other complications included minor bleeding $(n=2)$ and minor wound infection $(n=$ 9) which were treated conservatively.

\section{Outcome}

- Outcome analysed at 6 months.

- Patients available for follow-up- 45 .

- $\quad$ Primary graft patency at 6 months- 32 (64\%).

- $\quad$ Palpable pulse- 20.

- $\quad$ ABI improvement-12.

- Limb salvage rate with minor amputation- $80 \%$.

- No. of patients- 36 .

- Palpable pulse- 20.

- ABI improvement- 12 .

- Same ABI- 4

- Amputation rate- $20 \%(n=9)$ (Major).

- Of the 10 patients in whom the planned revascularisation was not surgically feasible, 5 patients reported for amputation at 3 - 6 months.

- $\quad$ SPSS software was used to analyse the statistics and to calculate the prevalence.



Figure 1. TAO Bypasses vs. Other Bypasses

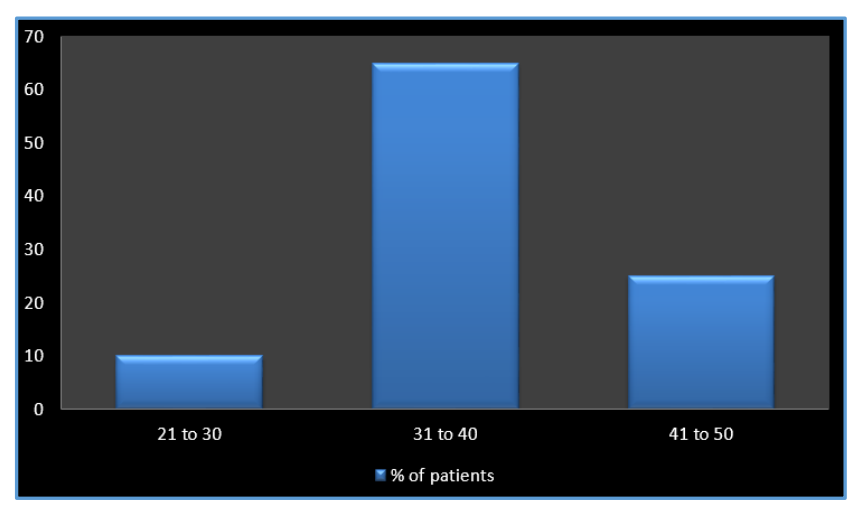

Figure 2. Age Distribution

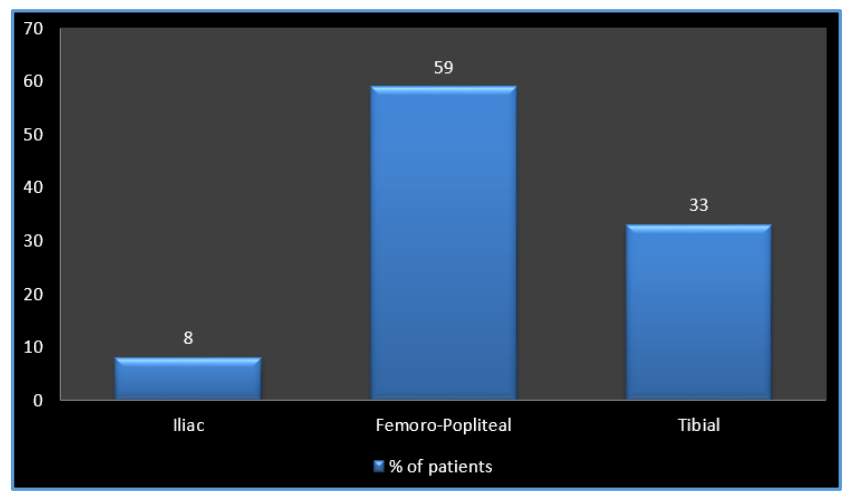

Figure 3. Level of Occlusion

\section{DISCUSSION}

TAO continues to be a common cause of peripheral arterial occlusive disease affecting predominantly young males in their most productive phase of their lives. These patients have a markedly diminished qualify of life. Tobacco cessation should be immediate, total and permanent to make any treatment useful in these patients. The treatment of those patients who continue to smoke not only renders treatment very difficult, but also results in treatment failure.

Most patients present very late in the clinical course with gangrene and non-salvageable limbs. Primary amputation is the only option in this group. Conservative line of management with medications is an option in those who present with nonrevascularisable vasculature as evidenced by CT angiograms. Surgical revascularisation is feasible only in select group of patients who constitute about $5-10 \%$ patients of TAO presenting with critical limb ischaemia. 
In our study with a probable diagnosis of TAO based on Shionoya criteria $^{7} 60$ patients have been chosen for revascularisation, since the patients had arteriographic evidence of a suitable distal bypassable vessel.

All patients in this study were male. This compares with the literature. Reports of TAO in women have been scarce.

The average age of all patients with TAO in this study was $35.8+/-7.2$ years and the average age of patients who underwent revascularisation was $37.1+/-7.04$ years. These patients are younger when compared to other series. The mean age in the series at the Cleveland Clinic was 42 years, while that in the Intractable Disease Research Committee report from Japan in 1993 was 50.8 years. In the series described by Shionoya, however, the average age at onset of disease was 35.8 years.

The data in this study have been calculated at the time of presentation to our institution, and since all our patients presented with critical limb ischaemia, the age of onset of symptoms would be comparable to that of Shionoya's series, but much younger than other series. Majority of patients with TAO in our study are in the 30 - 50 years' age group with the peak incidence in the $4^{\text {th }}$ decade. This is similar to the findings observed by Shionoya. In Shionoya series, the maximum incidence was noted in the fourth decade followed by the fifth, third and second decades. The youngest patient in our series was 23 years old and there were no patients in the second decade.

Majority of our patients presented with unilateral lower limb involvement. The incidence of bilateral lower limb involvement was $39.5 \%$. This is lower than in Shionoya's series where three or four limb involvement was higher at a staggering $83 \%$. In our study, $40 \%$ patients had bilateral lower limb ulcers or gangrene. Patients with bilateral critical limb ischaemia have been reported to have a higher incidence of recurrent ulcers/ gangrene and a higher amputation rate.

All the patients in our study were admitted with critical limb ischaemia. In Shionoya's series, $70 \%$ of patients presented to their institution with critical limb ischaemia as the first symptom. In view of the similarities between the two series, this could be true for our patients as well.

Majority of patients presented with gangrene $(77.6 \%)$ as compared to ulcers $(28.8 \%)$. The great toe was the most common site for gangrene as well as ulcers. This has been observed in the series published by Hiria et al too. This could possibly be attributed to the leading role played by the great toe in walking or exercise and susceptibility to external injury besides the almost solitary nutrient artery supplying great toethe first metatarsal artery. This was followed by other toes as the next common site for gangrene or ulceration, while the foot was the least common site.

Most patients had an antecedent history of lower limb intermittent claudication (72.6\%). Patients with tibial disease presented with foot claudication, while those with supragenicular involvement complained of calf claudication. The patients included in the study, i.e. those who underwent revascularisation had much higher incidence of previous lower limb claudication (87.6\%). Most of these patients presented with calf claudication, signifying the higher incidence of proximal vessel involvement. The low incidence of foot claudication could be attributable to the high incidence of necrotic foot lesions as a present symptom, which probably overshadow the foot claudication as a presenting symptom.
Disease progressing from the infrapopliteal to the suprapopliteal segment has been observed to cause calf claudication superseding the foot claudication.

This is as a result of the calf muscles working more actively during walking. In patients with improvement in calf claudication after revascularisation, foot claudication has been observed to re-emerge as a complaint, as the proximal vessels are more amenable to bypass while the crural disease remains non-revascularisable.

Of all the patients admitted with TAO, only $10 \%(n=60)$ were suitable candidates for revascularisation and limb salvage. The study by Sayin et al, ${ }^{8}$ Istanbul University shows arterial revascularisation in $9.7 \%$ patients with TAO. Of the sixty patients chosen for surgical revascularisation, surgical bypass was done only in 50 patients, while in ten patients the decision to carry out bypass had to be revised intraoperatively, because of distal outflow disease status and poor quality of autologous veins. This is an index of the higher rate of revascularisation attempt pursued at our centre. Similar findings have been reported by Dilege ${ }^{9}$ et al.

The level of occlusion on clinical examination correlated with the management outcome for the patients. The patients were clinically characterised as aortoiliac disease, unilateral iliac disease, femoropopliteal disease or tibial artery disease based on clinical pulse examination.

No patient had aortic disease in any of the groups studied. Involvement of the aorta has been reported very rarely in the literature. All series reflect the findings reported in our study (Ref: ${ }^{10}$ ).

Majority of limbs with iliac artery disease had a distal bypassable vessel, most commonly in the femoral arteries. All these limbs had segmental disease in the popliteal and tibial arteries and had a segment of artery fit for sequential bypass.

About 55\% of limbs with femoral artery occlusions had distal reformation of the bypassable vessel. This number though was much higher in the proximal femoral arterial disease and much lower with disease in the adductor hiatus. This lends credence to the hypothesis that the distal segments of the femoral artery are involved due to proximal progression of infrapopliteal disease and occur at a later stage of the disease, whereby the disease is more extensive and distal target vessels for bypass are scarce.

The proximal segments of the femoral arteries are probably involved by progression from disease in the muscular branches of the thigh or by skip lesion and occur at an earlier stage of the disease. The distal disease in this group tends to be less extensive and hence chances of finding a distal bypassable vessel are higher. This indicates the need to undertake revascularisation in this group of patients and a higher chance of limb salvages.

Patients with most proximal level of occlusion at the popliteal artery predominantly had disease in the middle and distal $1 / 3^{\text {rd }}$ of the artery. These patients had $100 \%$ incidence of disease in the tibial arteries. About one-third of these limbs, however, have distal reformation of a tibial artery, most commonly the anterior tibial artery. Collateralisation in this segment depends on the level of occlusion. The sural artery was the predominant collateral in occlusions below the knee, while the genicular arteries were important in suprageniculate occlusion. 
Collateralisation in crural artery occlusion has been described to be dependent on a patent peroneal artery, as communication between anterior and posterior tibial arteries is sparse. Occlusions of the posterior tibial artery have been noted to have the best collateralisation due to the larger number of branches from the posterior tibial artery and the larger muscle mass that is supplied.

Distal reformation and the presence of the bypassable vessel were closely related to the level of arterial occlusion. Hence, patients with proximal vessel disease have the highest chance of limb salvage through revascularisation.

Considering all these criteria, only a small proportion of TAO patients, around $10 \%$ qualify for surgical revascularisation. In these patients, especially with a proximal level of occlusion, attempts at revascularisation have been successful with a primary patency rate of $64 \%(n=32)$ at 6 months, of whom 20 patients had a palpable pulse and 12 patients showing ABI improvement. The study by Sayin ${ }^{2}$ and Associates reported primary patency rate of $63 \%$ at 5 years. Our follow-up has been 6 months and a primary patency rate $64 \%$ is expected to match the above reports at 2 years and 5 years. The limb salvage rate was about $80 \%$. The higher limb salvage rate over the graft patency rate is reflective of collateral network sustaining the limb vascularity.

Graft thrombectomy has been done in 4 patients with 2 patients sustaining graft patency, while in 2 patients the attempt failed.

Arterial revascularisation in TAO unlike ASO does not have many advocates, since its role has been deemed to be controversial. In a select category of TAO patients with a reformed tibial vessel, surgical revascularisation when performed does offer a good chance of limb salvage in these young patients. This study does however aim to provide information about the patterns of disease involvement and provides a guide to the patient's groups who need to be targeted for revascularisation aimed at limb salvages.

We ought to be neither too nihilistic, nor too optimistic. A rather pragmatic approach with regards to surgical revascularisation in critical limb ischaemia with TAO is attendant with good results and can mitigate the suffering of these unfortunate patients provided they comply with tobacco cessation.

\section{CONCLUSION}

- TAO continues to be a significant source of limb loss and morbidity.

- The mean age of all TAO patients with CLI was 35 years.

- $\quad$ All patients were male.

- Most patients presented with critical limb ischaemia.

- Two, three limb involvement was around 40\%.
- Angiographically, majority had femoropopliteal segment occlusion. Suprageniculate disease affects a significant proportion of patients with TAO in our population. All patients with suprageniculate disease have infrageniculate disease as well.

- $\quad$ Primary amputation is needed in 30\% patients with TAO.

- Revascularisation could be attempted in $10-15 \%$ patients with TAO.

- Suprapopliteal vessel disease is more amenable for revascularisation with the possibility of a suitable distal vessel unlike infrapopliteal disease, where suitable distal vessels for revascularisation are often absent.

- Pathogenetic mechanisms for initiation and progression of disease at different levels in the arterial tree are different. The different pathogenetic and anatomic factors impact the probability of revascularisation and limb salvage.

Further studies are required for accurate understanding of the aetiopathology of the disease and to evolve management strategies to reduce limb loss due to TAO. The disease is on the march, only the victim crawls. An attempt at revascularisation is a reaffirmation of our firm faith in man's inherent ability to leave his foot prints on the sands of time.

\section{REFERENCES}

[1] Olin JW. Thrombo Angitis Obliterans (Buerger's Disease). In: Rutherford RB, edr. Vascular surgery. $7^{\text {th }}$ edn. 2005: p. 404.

[2] Olin JW. Thrombo Angiitis Obliterans (Buerger's Disease). In Rutherford RB. Vascular Surgery. $7^{\text {th }}$ edn. 2005: p. 412-3.

[3] Olin JW. Thromboangitis obliterans (Buerger's disease). NEJM 2000;343(12):864-9.

[4] Shionoya S. Buerger's disease: pathology, diagnosis and treatment. University of Nagoya Press 1990: p. 29-30.

[5] Shionoya S. Buerger's disease: pathology, diagnosis and treatment. University of Nagoya Press 1990: p. 112.

[6] Shionoya S. Buerger's disease: pathology, diagnosis and treatment. University of Nagoya Press 1990: p. 108-9.

[7] Shionoya S. Buerger's disease: pathology, diagnosis and treatment. University of Nagoya Press 1990: p. 163-7.

[8] Sayin A, Bozkurt AK, Tuzun H, et al. Surgical treatment of Buerger's disease: experience with 216 patients. Cardiovasc Surg 1993;1(4):377-80.

[9] Dilege S, Aksoy M, Kayabali M, et al. Vascular reconstruction in Buerger's disease: Is it feasible? Surg Today 2002;32(12):1042-7.

[10] Kobayashi M, Nishikimi N, Komori K. Current pathological and clinical aspects of Buerger's disease in Japan. Ann Vasc Surg 2006;20(1):148-56. 